\title{
Sodium-glucose co-transporter 2 inhibition with empagliflozin improves cardiac function in rats after cardiac arrest
}

\section{Yunke Tan}

Sun Yat-Sen University

Kai Yu

Sun Yat-Sen University

Lian Liang

Sun Yat-Sen University

Yuanshan Liu

Sun Yat-Sen University

Fengqing Song

Sun Yat-Sen University

Qiulin Ge

Sun Yat-Sen University

Xiangshao Fang

Sun Yat-Sen University

Tao Yu

Sun Yat-Sen University

Zitong Huang

Sun Yat-Sen University

Longyuan Jiang

Sun Yat-Sen University

Peng Wang ( $\sim$ wangp49@mail.sysu.edu.cn )

Sun Yat-Sen University

\section{Original investigation}

Keywords: cardiac arrest, cardiopulmonary resuscitation, empagliflozin, mitochondria, ketone body

Posted Date: February 23rd, 2021

DOI: https://doi.org/10.21203/rs.3.rs-221519/v1 
License: (c) (i) This work is licensed under a Creative Commons Attribution 4.0 International License. Read Full License 


\section{Abstract}

Background: Sodium-glucose co-transporter 2 (SGLT2) inhibition reduces hyperglycaemia and has beneficial effects in heart failure. However, the effect of SGLT2 inhibition with empagliflozin on acute myocardial dysfunction after cardiac arrest (CA) remains unknown.

Methods: Non-diabetic male Sprague-Dawley rats underwent ventricular fibrillation to induce CA, or sham surgery. Rats received $10 \mathrm{mg} / \mathrm{kg}$ of empagliflozin or vehicle at 10 minutes after return of spontaneous circulation by intraperitoneal injection. Cardiac function was assessed by echocardiography, histological analysis, molecular markers of myocardial injury, oxidative stress, mitochondrial ultrastructural integrity and metabolism.

Results: Empagliflozin did not influence heart rate and blood pressure, but left ventricular function and survival time were significantly higher in the empagliflozin treated group compared to the group treated with vehicle. Empagliflozin also reduced myocardial contraction band necrosis, myocardial fibrosis, serum cardiac troponin I levels and myocardial oxidative stress after CA. Moreover, empagliflozin maintained the structural integrity of myocardial mitochondria and increased mitochondrial activity after CA. In addition, empagliflozin increased circulating and myocardial ketone levels as well as myocardial expression of the $\beta$-hydroxy butyrate dehydrogenase 1 . Together these metabolic changes were associated with an increase in cardiac ATP production.

Conclusions: Empagliflozin favorably affects cardiac function in non-diabetic rats with acute myocardial dysfunction after CA, associated with reducing glucose levels and increasing ketone body oxidized metabolism. Our data suggest that empagliflozin might be of benefit in patients with acute myocardial dysfunction after $\mathrm{CA}$.

\section{Background}

Cardiac arrest (CA) is an important public health challenge, and it seems to account for approximately $50 \%$ of all cardiovascular deaths [1]. A high-quality cardiopulmonary resuscitation (CPR) is essential to return of spontaneous circulation (ROSC) for CA victims. Patients who initially achieve ROSC after CA have high morbidity and mortality rates due to post-CA syndrome, including brain injury, myocardial dysfunction, and systemic inflammatory responses [2,3]. Post-CA myocardial dysfunction, including arterial hypotension, ventricular arrhythmias, and recurrent $\mathrm{CA}$ has been recognized as the leading cause of early death after ROSC $[3,4]$. Although therapeutic hypothermia has proved effective in clinical studies, no pharmacological treatment is available to improve outcomes of post-CA myocardial dysfunction [5].

Post-CA myocardial dysfunction is associated with global myocardial ischemia/reperfusion. It is widely acknowledged that ischemia/reperfusion leads to mitochondrial ultrastructural and functional injury. Main contributors to ischemia/reperfusion injury include ATP depletion and generation of reactive oxygen species (ROS) $[4,6]$. Recent studies aimed at protecting mitochondria from reperfusion injury during ROSC indicated that preservation of mitochondrial bioenergetic function in the myocardium helps 
restoration of cardiac activity and sustained post-resuscitation circulation [7, 8]. In addition, glucose homeostasis is important in the pathophysiology of critical illness and can be used as a determinant of outcome following resuscitation from CA. Hyperglycemia is commonly seen in patients after CA and is associated with worse outcomes [9-11]. Several clinical studies have suggested that treatment of hyperglycemia may improve morbidity and mortality in critically-ill patients $[12,13]$. However, some other studies have shown that tight glucose control through intensive insulin therapy may not necessarily improve outcomes in CA patients [14, 15].

Sodium glucose cotransporter 2 (SGLT2) is a channel protein primarily found in the proximal convoluted tubule of the kidney and regulates $90 \%$ of renal tubular glucose reabsorption using the gradient of the sodium ions concentration between inside and outside of the cells. Therefore, SGLT2 inhibitor can reduce glucose reabsorption and lower the blood glucose [16]. Empagliflozin, a highly selective SGLT2 inhibitor, is a newly developed oral antidiabetic drug to reduce hyperglycaemia in an insulin-independent manner. Beyond glucose control, recent studies found that SGLT2 inhibitor have a potential protective effect against cardiovascular events. Empagliflozin has demonstrated to reduce cardiovascular mortality by $38 \%$ and heart failure hospitalizations by $35 \%$ in patients with type 2 diabetes mellitus in the EMPA-REG OUTCOMES trial [17]. Furthermore, emerging evidence demonstrated the effectiveness of empagliflozin in non-diabetic heart failure [18-20]. The actual mechanisms responsible for these beneficial effects in heart failure are not completely clear. Thus far, a number of hypothesized mechanisms have been proposed to explain the cardioprotective effects of SGLT2 inhibitors, including beneficial effects on cardiac energy metabolism, reducing inflammation, blood pressure reduction, improving kidney function, decreasing oxidative stress, and so on [21]. However, the effect of empagliflozin on acute post-CA myocardial dysfunction and involved mechanism remain unknown.

In the present study, we therefore investigated the effects of empagliflozin on acute myocardial injury after CA and dissected the involved molecular mechanism in non-diabetic hearts. We hypothesized that empagliflozin could alleviate myocardial injury after CA by maintaining glucose homeostasis and protecting mitochondrial function. Here, we examined the effects of empagliflozin on acute myocardial injury in a non-diabetic rat model of ventricular fibrillation CA and CPR.

\section{Methods}

\section{Animals and drugs}

Male Sprague-Dawley rats (330-355 g) were obtained from Experimental Animal Center of Sun Yat-sen University (Guangzhou, China). Animals were kept under standard laboratory conditions in a specific pathogen-free room at a constant temperature $\left(20^{\circ} \mathrm{C}-22^{\circ} \mathrm{C}\right)$ with 12 hours of light/ 12 hours of dark exposure. All animal experiments were carried out in accordance with the ARRIVE guideline [22]. The experimental protocol was approved by the Institutional Animal Care and Use Committee of Sun Yat-sen University. 
Empagliflozin was purchased from MedChemExpress Inc. (Monmouth Junction, USA). Empagliflozin was dissolved in $10 \%$ dimethyl sulfoxide, $20 \%$ kolliphor (Sigma Aldrich, USA) and $80 \%$ phosphate buffer saline as a stock solution $(10 \mathrm{mg} / \mathrm{mL})$.

\section{CA model and experimental protocol}

The animals were surgically prepared as previously described [23]. CA was induced by ventricular fibrillation (VF) through a transoesophageal electrode. The electrode was positioned to ensure constant ventricular capture and was given a $30 \mathrm{~V}$ voltage and $30 \mathrm{~Hz}$ current. The current flow continued for 3 minutes to prevent a spontaneous reversal of VF. After 6 minutes of untreated VF, CPR was started with precordial compression (PC) at a rate of 250/minutes and mechanical ventilation with $100 \% \mathrm{O}_{2}$. After 2 minutes of PC, epinephrine $(0.01 \mathrm{mg} / \mathrm{kg})$ was administrated and $2 \mathrm{~J}$ biphasic waveform shocks was performed after 4 minutes of CPR. ROSC was defined as the return of supraventricular rhythm with a MAP $\geq 60 \mathrm{mmHg}$ for a minimum of 5 minutes. If VF persisted, another $2 \mathrm{~J}$ shock was given after another 1 minutes of PC. Resuscitation was declared a failure when there was no ROSC after 6 minutes of CPR. After ROSC, mechanical ventilation was continued with $100 \% \mathrm{O}_{2}$ at 30 minutes, $50 \% \mathrm{O}_{2}$ at 20 minutes, and $30 \% \mathrm{O}_{2}$ at 10 minutes.

After achieved ROSC, the animals were randomized into three groups: CA+ empagliflozin (EMP) group ( $n$ $=18), C A+$ vehicle group $(n=22)$ and Sham group. The animals in Sham group underwent the same operation with the other groups but without inducing CA. The rats in the CA + EMP group received 10 $\mathrm{mg} / \mathrm{kg}$ of empagliflozin at 10 minutes after ROSC by intraperitoneal injection. Meanwhile, the rats in Vehicle group received placebo (10\% dimethyl sulfoxide, $20 \%$ kolliphor and $80 \%$ phosphate buffer saline) for control. An illustration of the protocol used is provided (Fig. 1).

\section{Histopathologic analysis}

The heart tissues were embedded in paraffin and sectioned at $5 \mu \mathrm{m}$ thickness. Tissue slices of the hearts were stained with hematoxylin-eosin (HE) and Masson's trichrome (Servicebio, Guangzhou, China) for histological evaluation according to the manufacturer's instructions. Ten fields of vision (magnification of $\times 400$ ) were randomly sampled from each heart tissue sample under an optical microscope. The fibrotic tissue area stained blue (\%) by Masson staining was analyzed using counting software (Image J, National Institute of Health, USA).

\section{Serum cardiac troponin I (cTnl) levels}

Blood samples were collected from the abdominal aorta at 24 hours after ROSC in rats. The blood was allowed to clot for 30 minutes at room temperature. The clot was removed by centrifugation at $1500 \mathrm{~g}$ for 15 minutes and the serum was stored at $-80^{\circ} \mathrm{C}$ until use. Serum cTnl levels were detected using a rat cTnl enzyme-linked immunosorbent assay kit (Cusabio, China) according to the manufacturer's instructions.

\section{Echocardiography}


Rats were anesthetized with $2 \%$ to $3 \%$ isoflurane, and in vivo cardiac function was assessed by transthoracic echocardiography using a high-resolution imaging system equipped with a $30-\mathrm{MHz}$ transducer (Vevo 3100, VisualSonics, Toronto, Canada), as previously described [24]. The left ventricular

(LV) function was assessed by the ejection fraction (EF) and cardiac output (CO). All measurements were conducted by a single investigator who was blinded to the experimental groups.

\section{Oxidative stress analysis}

The oxidative stress in the heart tissue at 24 hours after CA was evaluated by immunohistochemical staining of 4-hydroxynonenal (4-HNE) to assess lipid peroxidation and 8-hydroxy-2'-deoxyguanosine (8$\mathrm{OHdG}$ ) to detect the extent of nucleic acid oxidation. Heart tissues were sectioned and embedded in paraffin. For immunohistochemistry, the fixed sections were immunostained overnight at $4^{\circ} \mathrm{C}$ using a primary monoclonal antibody against 4-HNE and 8-OHdG (Abcam, Cambridge, United Kingdom), followed by corresponding secondary antibody for $2.5 \mathrm{~h}$ at room temperature. Ten fields of vision (magnification of $\times 400$ ) were randomly sampled from each heart tissue sample under an optical microscope. 4-HNE and $8-\mathrm{OHdG}$ relative intensity area (\%) were detected by automated counting software (Image J, National Institute of Health, USA). The ROS levels in the heart at 6 hours after CA were measured using a tissue ROS assay kit (Genmed Scientifics, Wilmington, DE, USA) that utilized 2',7'-dichlorofluorescein diacetate as the oxidative fluorescent probe. The ROS signals were detected by a microplate reader (Molecular Devices, Sunnyvale, USA).

\section{Western blot analysis}

For immunoblotting, $\beta$-hydroxy butyrate dehydrogenase 1 (BDH1) (diluted 1:1000; Abcam, Cambridge, United Kingdom) and GAPDH (diluted 1:2000; Cell Signaling Technologies, USA) antibodies were used. Protein bands were detected using an enhanced chemiluminescence kit (Cell Signaling Technologies, USA). GAPDH was used to normalize protein loading. The densities of protein blots were quantified by using Image $\mathrm{J}$ software (National Institutes of Health, Bethesda, USA) and normalized to control.

\section{Transmission electron microscopy and analysis}

Heart samples were collected and fixed in $2.5 \%$ glutaraldehyde in $0.1 \mathrm{~mol} / \mathrm{L}$ phosphate buffer $(\mathrm{pH} 7.4)$ overnight at $4^{\circ} \mathrm{C}$. The heart tissues were cut into $500-\mu \mathrm{m}$ thick transverse slices and were post-fixed in $1 \%$ osmium tetroxide for 1 hour. Tissue slices were then dehydrated in ascending series of ethanol and embedded in epon. After dehydration, the specimens were conventionally processed and examined under a transmission electron microscope (Tecnai G2, FEl, Hillsboro, USA) through 13,500x objectives. Area of individual mitochondria was quantified by using Image $\mathrm{J}$ software (National Institutes of Health, Bethesda, USA).

\section{Mitochondrial complex $\square$ activity}

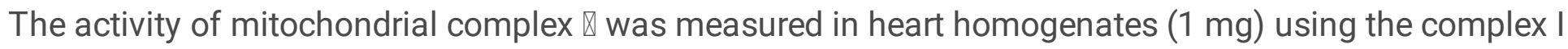
enzyme activity assay kit (Colorimetric) (Abcam, Cambridge, United Kingdom) according to the 
manufacturer's instructions.

\section{Measurement of metabolites levels}

ATP levels were measured in heart homogenates with an ATP bioluminescent assay kit (Beyotime, China) according to the manufacturer's instructions. $\beta$-hydroxybutyrate (BHB) concentrations in heart homogenates and serum were measured using a BHB colorimetric assay kit (Biovision, Milpitas, CA, USA) according to the manufacturer's instructions. Nicotinamide adenine dinucleotide $\left(N A D^{+}\right) /$reduced form of $\mathrm{NAD}^{+}(\mathrm{NADH})$ levels in heart homogenates were measured using a NAD ${ }^{+} / \mathrm{NADH}$ quantification colorimetric kit (Biovision, Milpitas, CA, USA) according to the manufacturer's instructions. Serum glucose and lactate concentrations were measured by a biochemistry analyzer (YSI, Yellow Springs, OH, USA).

\section{Statistical analysis}

All data were presented as mean \pm standard deviation (SD), and analyzed using GraphPad Prism 8 (GraphPad, San Diego, CA, USA). The unpaired t test was used to compare the same parameters between two groups. Normally distributed data were analyzed by one- or two-way analysis of variance (ANOVA) followed by Tukey's post hoc test. Lipid droplets number was analyzed by the Kruskal-Wallis test with Dunn's post hoc analyses between multiple groups. The survival curve among the groups was obtained using Kaplan-Meier survival analysis and compared with a log-rank test. A two-tailed $P<0.05$ was considered significant.

\section{Results}

\section{Animal physiologic data and resuscitation characteristics}

A total of 52 rats were prepared for this study. Of these rats, 6 rats received sham operation (The Sham group), and 46 other rats underwent CPR but 6 rats failed to achieve ROSC. In the CA + vehicle group, 22 rats achieved ROSC but 10 rats failed to survive at measurement time point. In the CA + EMP group, 18 rats achieved ROSC but 6 rats failed to survive at measurement time point. The rats that failed to achieve ROSC were excluded from the group. There were no statistically significant differences in baseline physiologies, hemodynamic, cardiac function, and blood analytical measurements among three groups (Table 1). For rats that achieved ROSC, the time of CPR was comparative between the CA + vehicle and CA + EMP group (Table 1).

\section{Effect of empagliflozin on cardiac function after CA}

To explore the effect of empagliflozin on hemodynamic in rats, heart rate and blood pressure were detected at 15, 30 and 60 minutes after CA. Our results showed that both heart rate (Fig. 2a) and blood pressure (Fig. 2b) were significantly decreased within 1 hour after ROSC $(P<0.01)$. Empagliflozin treatment had no effects on heart rate (Fig. 2a) and blood pressure (Fig. 2b) within 1 hour after CA. In addition, the cardiac function was measured by Doppler echocardiography at 24 hours after CA. We 
found that LVEF (Fig. 2c) and CO (Fig. 2d) were significantly decreased after CA $(P<0.01)$. However, empagliflozin treatment significantly increased both LVEF and CO (Fig. 2c, d) (LVEF: $71.7 \pm 7.1$ versus $51.7 \pm 9.3, P<0.01 ; C 0: 55.2 \pm 5.8$ versus $38.7 \pm 5.7, P<0.01)$. Moreover, Kaplan-Meier survival analysis showed that empagliflozin treatment significantly increased the survival time of the animals after $C A$ within 24 hours $(P<0.05)$ (Fig. 2e).

\section{Effect of empagliflozin on myocardial injury after CA}

Histological analysis with HE and Masson staining were performed to detect the myocardial injury at 24 hours after CA. HE staining exhibited contraction band necrosis in the myocardium after CA, whereas there was few myocardial contraction band necrosis after empagliflozin treatment (Fig. 3a). Compared with the sham group, the myocardial fibrosis significantly increased in the CA + vehicle group (Fig. 3a). However, the myocardial fibrosis was significantly lower in the CA + EMP group than that in the CA + vehicle group ( $6 \% \pm 2 \%$ versus $10 \% \pm 3 \%, P<0.05)$ (Fig. $3 \mathrm{~b}$ ). In addition, serum cTnl levels were detected in three groups at 24 hours after CA. The serum cTnI concentration increased significantly after ROSC $(P$ $<0.01)$. However, the serum cTnl concentration in the CA + EMP group was significantly decreased compared with the $\mathrm{CA}+$ vehicle group $(15 \pm 4 \mathrm{pg} / \mathrm{mL}$ versus $20 \pm 4 \mathrm{pg} / \mathrm{mL})(P<0.05)$ (Fig. 3c). These results indicated that empagliflozin treatment decreased myocardial pathohistological changes after $\mathrm{CA}$ in rats.

\section{Effect of empagliflozin on oxidative stress after CA}

Immunohistochemistry for 4-HNE (the end product of lipid peroxidation) and 8-OHdG (an index of oxidative DNA damage) was carried out to investigate oxidative stress in myocardium at 24 hours after $\mathrm{CA}$. The 4-HNE positive cardiomyocytes and 8-OHdG positive cells were distributed throughout the myocardium after CA (Fig. 4a). Notably, there were fewer 4-HNE positive cardiomyocytes and 8-OHdG positive cells obtained from the rats administered empagliflozin (4-HNE: $52 \% \pm 13 \%$ versus $70 \% \pm 10 \%, P$ $<0.05$; 8-OHdG: $30 \% \pm 9 \%$ versus $52 \% \pm 11 \%, P<0.01$ ) (Fig. 4b, c). Moreover, the myocardial ROS levels were detected in three groups at 24 hours after $\mathrm{CA}$. The myocardial ROS levels increased significantly after ROSC $(P<0.01)$. However, the myocardial ROS levels in the CA + EMP group were significantly decreased compared with the $\mathrm{CA}+$ vehicle group $(1.6 \pm 0.3$ versus $2.9 \pm 0.5, P<0.01)$ (Fig. $4 \mathrm{~d}$ ). These results indicated that empagliflozin treatment ameliorated oxidative myocardial injury after CA in rats.

\section{Effect of empagliflozin on mitochondrial ultrastructural integrity after CA}

The structure and integrity of mitochondria are essential for the physiological function of the heart. To determine the effect of empagliflozin on mitochondrial ultrastructure after CA, the myocardial tissue was examined at 24 hours after CA by transmission electron microscopy. The electron microscopy analysis showed that mitochondria appeared smaller in the $\mathrm{CA}+$ vehicle group compared with mitochondria from the sham group, suggesting mitochondrial fission after CA (Fig. 5a). However, empagliflozin treatment significantly decreased mitochondrial fission after $C A$ (mitochondrial area: $0.8 \pm 0.3 \mu \mathrm{m}^{2}$ versus $0.6 \pm 0.4$ $\mu \mathrm{m}^{2}, P<0.01$ ) (Fig. 5b). In addition, the total number of lipid droplets in cardiomyocytes was significantly 
increased after CA. However, empagliflozin treatment significantly decreased the total number of lipid droplets after CA $(2.5 \pm 1.4$ versus $3.6 \pm 1.8, P<0.05)$ (Fig. $5 \mathrm{c})$. The number of intermitochondrial junctions (IMJs) is an index of highly active mitochondria [25]. Our results found that the number of IMJs in cardiomyocytes was significantly decreased after CA, but empagliflozin treatment significantly increased the number of IMJs after CA (1.6 \pm 0.4 versus $1.3 \pm 0.4, P<0.05)$ (Fig. $5 d$ ). These results demonstrated that empagliflozin treatment maintained the structural integrity of myocardial mitochondria after CA.

\section{Effect of empagliflozin on myocardial energy metabolism after CA}

Alterations in mitochondrial bioenergetics play a key role in heart failure $[26,27]$. Our results found that the myocardial ATP levels were significantly decreased at 6 and 24 hours after ROSC, but empagliflozin treatment significantly increased the myocardial ATP levels at 6 and 24 hours after ROSC ( 6 hours: $80 \pm$ $9 \%$ versus $62 \pm 9 \%, P<0.01 ; 24$ hours: $92 \pm 7 \%$ versus $76 \pm 9 \%, P<0.01$ ) (Fig. 6a). We next investigated whether empagliflozin could affect glucose metabolism after resuscitation. The results showed that serum glucose levels were significantly increased at 6 and 24 hours after ROSC, respectively. However, empagliflozin treatment significantly decreased serum glucose levels at 6 and 24 hours (6 hours: $8.3 \pm$ $1.3 \mathrm{mmol} / \mathrm{L}$ versus $12.4 \pm 2.7 \mathrm{mmol} / \mathrm{L}, P<0.01 ; 24$ hours: $7.2 \pm 0.9 \mathrm{mmol} / \mathrm{L}$ versus $9.3 \pm 1.5 \mathrm{mmol} / \mathrm{L}, P<$ 0.05 ) (Fig. 6b). In addition, empagliflozin treatment significantly decreased serum lactate levels at 6 and 24 hours (6 hours: $3.7 \pm 1.0 \mathrm{mmol} / \mathrm{L}$ versus $5.5 \pm 0.9 \mathrm{mmol} / \mathrm{L}, P<0.01 ; 24$ hours: $2.4 \pm 0.7 \mathrm{mmol} / \mathrm{L}$ versus $3.4 \pm 0.6 \mathrm{mmol} / \mathrm{L}, P<0.05$ ) (Fig. $6 \mathrm{c}$ ). These results suggested that empagliflozin treatment increased myocardial energy production independent of glucose metabolism after CA.

\section{Effects of empagliflozin on myocardial ketone metabolism and mitochondrial activity after CA}

Ketones are an important alternative fuel for the cell under glucose hypometabolic conditions. To determine the role of ketones in empagliflozin mediated energy metabolism, BHB concentrations were detected in the serum and heart at 6 and 24 hours after CA. The serum BHB concentrations were significantly increased after empagliflozin treatment at 6 and 24 hours after ROSC, respectively ( 6 hours: $0.38 \pm 0.07 \mathrm{mmol} / \mathrm{L}$ versus $0.31 \pm 0.07 \mathrm{mmol} / \mathrm{L}, P<0.05 ; 24$ hours: $0.55 \pm 0.08 \mathrm{mmol} / \mathrm{L}$ versus $0.36 \pm$ $0.04 \mathrm{mmol} / \mathrm{L}, P<0.01$ ) (Fig. 7a). Similarly, empagliflozin treatment significantly increased the myocardial BHB concentrations at 6 and 24 hours after ROSC, respectively ( 6 hours: $2.26 \pm 0.41 \mathrm{mmol} / \mathrm{L}$ versus 1.73 $\pm 0.36 \mathrm{mmol} / \mathrm{L}, P<0.05 ; 24$ hours: $2.95 \pm 0.51 \mathrm{mmol} / \mathrm{L}$ versus $2.00 \pm 0.32 \mathrm{mmol} / \mathrm{L}, P<0.01$ ) (Fig. $7 \mathrm{~b}$ ). To determine whether the increases in ketone levels was associated with changes in the myocardial capacity to utilize ketone bodies, a critical protein named BDH1 involved in myocardial ketolysis was detected. The protein expression of $\mathrm{BDH} 1$ was significantly increased after empagliflozin treatment at 24 hours after ROSC $(P<0.05)$ (Fig. 7c, d). Maintenance of an optimal NAD ${ }^{+} / \mathrm{NADH}$ ratio is essential for mitochondrial function [28]. Our data showed that the myocardial $\mathrm{NAD}^{+} / \mathrm{NADH}$ ratios were significantly increased after empagliflozin treatment at 6 and 24 hours after ROSC, respectively ( 6 hours: $2.6 \pm 0.4$ versus $1.9 \pm 0.4, P$ $<0.01$; 24 hours: $2.8 \pm 0.5$ versus $2.4 \pm 0.4 \mathrm{mmol} / \mathrm{L}, P<0.05$ ) (Fig. $7 \mathrm{e}$ ). Moreover, we also found that empagliflozin treatment significantly increased the myocardial mitochondrial complex》activity at 6 and 
24 hours after ROSC, respectively ( 6 hours: $79 \pm 9 \%$ versus $60 \pm 11 \%, P<0.05$; 24 hours: $85 \pm 7 \%$ versus $72 \pm 9 \%, P<0.05$ ) (Fig. 7f). Taken together, these results demonstrated that empagliflozin treatment increased the myocardial ketone metabolism and mitochondrial function after CA.

\section{Discussion}

Empagliflozin is a newly approved antidiabetic drug that inhibits SGLT2 in an insulin-independent manner. In addition to its excellent glucose-lowering effect, recent studies demonstrated that empagliflozin markedly improved myocardial function in diabetic and non-diabetic heart failure [17-20]. However, whether empagliflozin improves cardiac outcomes in acute myocardial injury after CA is unknown. In the present study, we investigated the effects of empagliflozin on myocardial injury in rats using a VF induced CA and CPR model. Maintaining hemodynamics and preventing circulatory failure are important for early postresuscitation care [29]. Although empagliflozin did not improve the heart rate and blood pressure after resuscitation, it did not worsen the heart hemodynamics within 1 hour after resuscitation in our results. We found that empagliflozin treatment significantly increased the cardiac LVEF, CO and survival time of the animals at 24 hours after CA. In addition, empagliflozin decreased myocardial fibrosis and serum cTnl levels after resuscitation. Therefore, our findings suggested that empagliflozin had a protective effect on acute myocardial injury after CA in non-diabetic rats.

It has long been recognized that reoxygenation of myocardial tissue results in an increase in the production of oxidative stress due to excess ROS $[4,6]$. The mitochondria are major sources of ROS within the cell and as an important target for the functional disruption of the cell stimulated by ROS. The uncontrolled of mitochondria upon reoxygenation with subsequent ROS generation has deleterious effects on numerous macromolecules, such as proteins, lipids and DNA, leading to impaired cell function. ROS can lead to DNA damage and formation of 8-OHdG, a prominent feature in diabetic hearts [30]. Lipid oxidation by ROS results in formation of carbonyl compounds, such as 4-HNE, toxic metabolites that can promote numerous pathologies. The detrimental ROS mediates myocardial injury and cardiomyocyte death through a number of different mechanisms. Damaged mitochondria result in enhanced ROS production and activation of the NLRP3 inflammasome, which may promote or exacerbate cardiac fibrosis [31]. Moreover, ROS can modulate sarcomere function by affecting key proteins forming the thick and thin filaments [32]. Our study showed that 4-HNE and 8-OHdG positive cardiomyocytes were significantly increased after ROSC, indicating high oxidative stress in heart. However, empagliflozin treatment ameliorated oxidative myocardial injury after CA. Serval studies have demonstrated that hyperglycemia-induced pathogenic mechanisms contributed to ROS production, such as glucose autooxidation, metabolism, and formation of advanced glycosylation end products [33, 34]. Given that hyperglycemia occurs after $\mathrm{CA}$, the effect of empagliflozin on myocardial oxidative stress after CA may be associated with reduction of glucose level. Furthermore, it has reported that empagliflozin suppressed myocardial oxidative stress through activation of Nrf2/ARE signaling in diabetic mice [35].

Mitochondria are remarkable organelles capable of remodeling their structural organization to optimize mitochondrial function [36]. In our study, we found that the structure and integrity of mitochondria in 
heart were changed after CA such as elevation of mitochondrial fission and the number of lipid droplets, and empagliflozin treatment maintained the structural integrity of myocardial mitochondria after CA. Mitochondrial quality control mechanisms are largely regulated by mitochondrial dynamics, including fission (division) and fusion. Mitochondrial fission creates smaller, more discrete mitochondria, which are more capable of generating ROS and leads to cell apoptosis. Previous studies have demonstrated that inhibition of mitochondrial fission protected the heart against ischemia/reperfusion injury [37, 38]. Lipid droplets are cytosolic organelles composed of fatty acids. Mitochondria-lipid droplet interactions represent a significant mitochondrial network structure, and these interactions are dynamic and the frequency. The function of mitochondria-lipid interactions may support either metabolism or synthesis of fatty acids depending on the cell type and physiology of the cells [36]. Overabundant and/or enlarged lipid droplets are the hallmarks of obesity, type 2 diabetes, cardiac steatosis, and cardiomyopathy [39]. It has reported that accumulation of lipids in myocardial lipid droplets was associated with heart failure in obesity and diabetes mellitus [40]. Hyperglycemia may play an important role in mitochondrial dynamic and lipid droplets accumulation. It has reported that hyperglycemia enhances the ischemia-induced mitochondrial fission by ROCK1 activation and Drp1 translocation to the mitochondria [41]. Furthermore, study showed that high glucose concentration increases the amount of lipid droplets on colorectal cells [42].

Alterations in mitochondrial energy metabolism are common in many forms of heart disease, including heart failure, ischemic heart disease and diabetic cardiomyopathies [43]. In healthy heart, $60 \%$ to $90 \%$ of ATP production comes from $\beta$-oxidation of fatty acids, whereas $10 \%$ to $40 \%$ comes from oxidation of pyruvate derived from glycolysis [6]. Fatty acids are the major source of myocardial energy metabolism but have high oxygen requirements. During myocardial ischemia, glucose becomes the preferred myocardial energy metabolism substrate because it is more oxygen efficient than fatty acids, but this is at the expense of lower energy produced $[27,43]$. At that time, pyruvate produced by glycolysis is not oxidized via oxidative phosphorylation in mitochondria, but rather is reduced to lactate in the cytosol. The increase in glycolytic rate results in anaerobic production of ATP and excess cytosolic protons, leads to intracellular acidosis. The increase in lactate and proton production has the potential to be detrimental to the heart. The serum lactate level is a good prognostic predictor for CA patients and elevation of lactate concentration has been associated with increased mortality after out-of-hospital CA [44]. Therefore, the shift from in myocardial energy metabolism from fatty acids to glucose consumption lead to an energy deficit and lactate accumulation that impairs cardiac efficiency. Thus, a reduction of ATP level, decrease of mitochondrial complex囚activity, and accumulation of lipid droplets and lactate were observed in heart after CA in our study. Ketone bodies are an alternative fuel when energy is insufficient, and are more efficient than fatty acids on the basis of ATP produced per oxygen consumed (P/O ratio) [45]. Moreover, ketone bodies as a substrate for mitochondrial energy metabolism have no lactate production. Our study found that empagliflozin treatment significantly increased myocardial ATP levels, ketone bodies and mitochondrial activity after CA. Several recent studies have demonstrated that ketone body metabolism significantly protected the failing heart $[45,46]$. Therefore, our results suggested that the protective effects of empagliflozin on myocardial dysfunction after CA were mediated by ketone body metabolism. 
We acknowledged that our study had some limitations. First, we did not include an empagliflozin-treated sham group because our primary focus was the effects of empagliflozin on myocardial dysfunction after CA. Second, our study focused on the left ventricular systolic function after CA, but the diastolic characteristics of post-CA myocardial dysfunction were not defined. In future studies, the effect of empagliflozin on diastolic function of the heart after CA would be examined. Third, our study mainly focused on the early stage of myocardial dysfunction ( 6 and 24 hours of CA) and the associated pathological processes after CA. Future studies should prolong the observation period to assess survival and outcomes. Finally, we used only adult male rats for animal model in our study, but CA also occurs in both elderly males and females clinically. Future studies should be investigated in aged and female rats to better simulate the human condition.

\section{Conclusions}

Our data demonstrated that SGLT2 inhibition with empagliflozin ameliorated myocardial fibrosis, enhanced left ventricular function, and increased survival time in a non-diabetic rat model ventricular fibrillation CA and CPR. These cardiac benefits of empagliflozin seem to be mediated by reducing glucose levels and increasing ketone body oxidized metabolism, which improves myocardial energetics and cardiac function. Our findings highlight the therapeutic potential of empagliflozin for acute myocardial dysfunction after $\mathrm{CA}$ and warrant further investigation.

\section{Declarations}

\section{Acknowledgements}

Not applicable.

\section{Authors' contributions}

PW and LJ conceived the study and participated in its design and coordination. YT, KY and LL performed the experiments and drafted the manuscript. YL, FS, QG and XF assisted with the data collection. TY and $\mathrm{ZH}$ contributed to data analysis and interpretation. All authors read and approved the final manuscript.

\section{Funding}

This study was supported by the National Natural Science Foundation of China (No. 81971805), Guangdong Basic and Applied Basic Research Foundation (No. 2020A1515010158).

\section{Availability of data and materials}

The datasets used and/or analyzed during the current study are available from the corresponding author upon reasonable request.

\section{Ethics approval and consent to participate}


The study complied with the Declaration of Helsinki and was approved by the Institutional Animal Care and Use Committee of Sun Yat-sen University.

\section{Consent for publication}

Not applicable.

\section{Competing interests}

The authors have no conflicts of interest to declare.

\section{Abbreviations}

CA: cardiac arrest; CPR: cardiopulmonary resuscitation; ROSC: return of spontaneous circulation; ROS: reactive oxygen species; SGLT2: sodium glucose cotransporter 2; VF: ventricular fibrillation; PC: precordial compression; EMP: empagliflozin; HE: hematoxylin-eosin; cTnl: cardiac troponin l; LV: left ventricular; EF: ejection fraction; CO: cardiac output; 4-HNE: 4-hydroxynonenal; 8-OHdG: 8-hydroxy-2'-deoxyguanosine; $\mathrm{BDH} 1$ : $\beta$-hydroxy butyrate dehydrogenase 1 ; BHB: $\beta$-hydroxybutyrate; $\mathrm{NAD}^{+}$: nicotinamide adenine dinucleotide; SD: standard deviation; IMJs: intermitochondrial junctions.

\section{References}

1. Fishman GI, Chugh SS, Dimarco JP, Albert CM, Anderson ME, Bonow RO, et al. Sudden cardiac death prediction and prevention: report from a National Heart, Lung, and Blood Institute and Heart Rhythm Society Workshop. Circulation. 2010;122(22):2335-48.

2. Girotra S, Nallamothu BK, Spertus JA, Li Y, Krumholz HM, Chan PS. American Heart Association Get with the Guidelines-Resuscitation I. Trends in survival after in-hospital cardiac arrest. N Engl J Med. 2012;367(20):1912-20.

3. Nolan JP, Neumar RW, Adrie C, Aibiki M, Berg RA, Bottiger BW, et al. Post-cardiac arrest syndrome: epidemiology, pathophysiology, treatment, and prognostication. A Scientific Statement from the International Liaison Committee on Resuscitation; the American Heart Association Emergency Cardiovascular Care Committee; the Council on Cardiovascular Surgery and Anesthesia; the Council on Cardiopulmonary, Perioperative, and Critical Care; the Council on Clinical Cardiology; the Council on Stroke. Resuscitation. 2008;79(3):350-79.

4. Patil KD, Halperin HR, Becker LB. Cardiac arrest: resuscitation and reperfusion. Circ Res. 2015;116(12):2041-49.

5. Hypothermia after Cardiac Arrest Study G. Mild therapeutic hypothermia to improve the neurologic outcome after cardiac arrest. N Engl J Med. 2002;346(8):549-56.

6. Yang KC, Kyle JW, Makielski JC, Dudley SC, Jr. Mechanisms of sudden cardiac death: oxidants and metabolism. Circ Res. 2015;116(12):1937-55. 
7. Ayoub IM, Radhakrishnan J, Gazmuri RJ. Targeting mitochondria for resuscitation from cardiac arrest. Crit Care Med. 2008;36(11 Suppl):S440-6.

8. Gazmuri RJ, Radhakrishnan J. Protecting mitochondrial bioenergetic function during resuscitation from cardiac arrest. Crit Care Clin. 2012;28(2):245-70.

9. Ettleson MD, Arguello V, Wallia A, Arguelles L, Bernstein RA, Molitch ME. Hyperglycemia and insulin resistance in cardiac arrest patients treated with moderate hypothermia. J Clin Endocrinol Metab. 2014;99(10):E2010-14.

10. Beiser DG, Carr GE, Edelson DP, Peberdy MA, Hoek TL. Derangements in blood glucose following initial resuscitation from in-hospital cardiac arrest: a report from the national registry of cardiopulmonary resuscitation. Resuscitation. 2009;80(6):624-30.

11. Nurmi J, Boyd J, Anttalainen N, Westerbacka J, Kuisma M. Early increase in blood glucose in patients resuscitated from out-of-hospital ventricular fibrillation predicts poor outcome. Diabetes Care. 2012;35(3):510-12.

12. Van den Berghe G, Wilmer A, Hermans G, Meersseman W, Wouters PJ, Milants I, et al. Intensive insulin therapy in the medical ICU. N Engl J Med. 2006;354(5):449-61.

13. Vogt JA, Wachter U, Wagner K, Calzia E, Groger M, Weber S, et al. Effects of glycemic control on glucose utilization and mitochondrial respiration during resuscitated murine septic shock. Intensive Care Med Exp. 2014;2(1):19.

14. Losert H, Sterz F, Roine RO, Holzer M, Martens P, Cerchiari E, et al. Strict normoglycaemic blood glucose levels in the therapeutic management of patients within $12 \mathrm{~h}$ after cardiac arrest might not be necessary. Resuscitation. 2008;76(2):214-20.

15. Oksanen T, Skrifvars MB, Varpula T, Kuitunen A, Pettila V, Nurmi J, et al. Strict versus moderate glucose control after resuscitation from ventricular fibrillation. Intensive Care Med. 2007;33(12):2093-100.

16. de Leeuw AE, de Boer RA. Sodium-glucose cotransporter 2 inhibition: cardioprotection by treating diabetes-a translational viewpoint explaining its potential salutary effects. Eur Heart J Cardiovasc Pharmacother. 2016;2(4):244-55.

17. Fitchett D, Zinman B, Wanner C, Lachin JM, Hantel S, Salsali A, et al. Heart failure outcomes with empagliflozin in patients with type 2 diabetes at high cardiovascular risk: results of the EMPA-REG OUTCOME(R) trial. Eur Heart J. 2016;37(19):1526-34.

18. Packer M, Anker SD, Butler J, Filippatos G, Pocock SJ, Carson P, et al. Cardiovascular and Renal Outcomes with Empagliflozin in Heart Failure. N Engl J Med. 2020;383(15):1413-24.

19. Yurista SR, Sillje HHW, Oberdorf-Maass SU, Schouten EM, Pavez Giani MG, Hillebrands JL, et al. Sodium-glucose co-transporter 2 inhibition with empagliflozin improves cardiac function in nondiabetic rats with left ventricular dysfunction after myocardial infarction. Eur $\mathrm{J}$ Heart Fail. 2019;21(7):862-73.

20. Santos-Gallego CG, Requena-Ibanez JA, San Antonio R, Ishikawa K, Watanabe S, Picatoste B, et al. Empagliflozin Ameliorates Adverse Left Ventricular Remodeling in Nondiabetic Heart Failure by 
Enhancing Myocardial Energetics. J Am Coll Cardiol. 2019;73(15):1931-44.

21. Lopaschuk GD, Verma S. Mechanisms of Cardiovascular Benefits of Sodium Glucose Co-Transporter 2 (SGLT2) Inhibitors: A State-of-the-Art Review. JACC Basic Transl Sci. 2020;5(6):632-44.

22. Kilkenny C, Browne WJ, Cuthill IC, Emerson M, Altman DG. Improving bioscience research reporting: the ARRIVE guidelines for reporting animal research. PLoS Biol. 2010;8(6):e1000412.

23. Wang P, Chen M, Yang Z, Yu T, Zhu J, Zhou L, et al. Activation of Pyruvate Dehydrogenase Activity by Dichloroacetate Improves Survival and Neurologic Outcomes After Cardiac Arrest in Rats. Shock. 2018;49(6):704-11.

24. Yin M, Sillje HH, Meissner M, van Gilst WH, de Boer RA. Early and late effects of the DPP-4 inhibitor vildagliptin in a rat model of post-myocardial infarction heart failure. Cardiovasc Diabetol. 2011;10:85.

25. Picard M, McManus MJ, Csordas G, Varnai P, Dorn GW, Williams D, et al. Trans-mitochondrial coordination of cristae at regulated membrane junctions. Nat Commun. 2015;6:6259.

26. Ardehali H, Sabbah HN, Burke MA, Sarma S, Liu PP, Cleland JG, et al. Targeting myocardial substrate metabolism in heart failure: potential for new therapies. Eur J Heart Fail. 2012;14(2):120-29.

27. Fillmore N, Lopaschuk GD. Targeting mitochondrial oxidative metabolism as an approach to treat heart failure. Biochim Biophys Acta. 2013;1833(4):857-65.

28. Stein LR, Imai S. The dynamic regulation of NAD metabolism in mitochondria. Trends Endocrinol Metab. 2012;23(9):420-28.

29. Chang WT, Ma MH, Chien KL, Huang CH, Tsai MS, Shih FY, et al. Postresuscitation myocardial dysfunction: correlated factors and prognostic implications. Intensive Care Med. 2007;33(1):88-95.

30. Nishio S, Teshima Y, Takahashi N, Thuc LC, Saito S, Fukui A, et al. Activation of CaMKII as a key regulator of reactive oxygen species production in diabetic rat heart. $\mathrm{J}$ Mol Cell Cardiol. 2012;52(5):1103-11.

31. Zhou R, Yazdi AS, Menu P, Tschopp J. A role for mitochondria in NLRP3 inflammasome activation. Nature. 2011;469(7329):221-25.

32. Santos CX, Anilkumar N, Zhang M, Brewer AC, Shah AM. Redox signaling in cardiac myocytes. Free Radic Biol Med. 2011;50(7):777-93.

33. Kaludercic N, Di Lisa F. Mitochondrial ROS Formation in the Pathogenesis of Diabetic Cardiomyopathy. Front Cardiovasc Med. 2020;7:12.

34. Lu S, Liao Z, Lu X, Katschinski DM, Mercola M, Chen J, et al. Hyperglycemia Acutely Increases Cytosolic Reactive Oxygen Species via O-linked GIcNAcylation and CaMKII Activation in Mouse Ventricular Myocytes. Circ Res. 2020;126(10):e80-e96.

35. Li C, Zhang J, Xue M, Li X, Han F, Liu X, et al. SGLT2 inhibition with empagliflozin attenuates myocardial oxidative stress and fibrosis in diabetic mice heart. Cardiovasc Diabetol. 2019;18(1):15.

36. Glancy B, Kim Y, Katti P, Willingham TB. The Functional Impact of Mitochondrial Structure Across Subcellular Scales. Front Physiol. 2020;11:541040. 
37. Ong SB, Subrayan S, Lim SY, Yellon DM, Davidson SM, Hausenloy DJ. Inhibiting mitochondrial fission protects the heart against ischemia/reperfusion injury. Circulation. 2010;121(18):2012-22.

38. Sharp WW, Beiser DG, Fang YH, Han M, Piao L, Varughese J, et al. Inhibition of the mitochondrial fission protein dynamin-related protein 1 improves survival in a murine cardiac arrest model. Crit Care Med. 2015;43(2):e38-47.

39. Krahmer N, Farese RV, Jr., Walther TC. Balancing the fat: lipid droplets and human disease. EMBO Mol Med. 2013;5(7):973-83.

40. Sharma S, Adrogue JV, Golfman L, Uray I, Lemm J, Youker K, et al. Intramyocardial lipid accumulation in the failing human heart resembles the lipotoxic rat heart. FASEB J. 2004;18(14):1692-700.

41. Wang W, Wang Y, Long J, Wang J, Haudek SB, Overbeek P, et al. Mitochondrial fission triggered by hyperglycemia is mediated by ROCK1 activation in podocytes and endothelial cells. Cell Metab. 2012;15(2):186-200.

42. Tirinato L, Pagliari F, Di Franco S, Sogne E, Marafioti MG, Jansen J, et al. ROS and Lipid Droplet accumulation induced by high glucose exposure in healthy colon and Colorectal Cancer Stem Cells. Genes Dis. 2020;7(4):620-35.

43. Fillmore N, Mori J, Lopaschuk GD. Mitochondrial fatty acid oxidation alterations in heart failure, ischaemic heart disease and diabetic cardiomyopathy. Br J Pharmacol. 2014;171(8):2080-90.

44. Shinozaki K, Oda S, Sadahiro T, Nakamura M, Hirayama Y, Watanabe E, et al. Blood ammonia and lactate levels on hospital arrival as a predictive biomarker in patients with out-of-hospital cardiac arrest. Resuscitation. 2011;82(4):404-9.

45. Lopaschuk GD, Karwi QG, Ho KL, Pherwani S, Ketema EB. Ketone metabolism in the failing heart. Biochim Biophys Acta Mol Cell Biol Lipids. 2020;1865(12):158813.

46. Schulze PC, Wu JMF. Ketone bodies for the starving heart. Nat Metab. 2020;2(11):1183-85.

\section{Tables}




\begin{tabular}{llll}
\hline Variables & $\begin{array}{l}\text { Sham } \\
(\mathbf{n}=6)\end{array}$ & $\begin{array}{l}\text { CA+vehicle } \\
(\mathbf{n}=22)\end{array}$ & $\begin{array}{l}\text { CA+EMP } \\
(\mathbf{n}=18)\end{array}$ \\
\hline Weight $(\mathrm{g} \square$ & $340 \pm 10$ & $342 \pm 12$ & $344 \pm 11$ \\
Heart rate (beats/min) & $386 \pm 21$ & $379 \pm 25$ & $383 \pm 27$ \\
\hline MAP $(\mathrm{mm} \mathrm{Hg})$ & $132 \pm 6$ & $136 \pm 4$ & $133 \pm 6$ \\
\hline Rectal temperature $\left({ }^{\circ} \mathrm{C}\right)$ & $36.4 \pm 0.3$ & $36.7 \pm 0.4$ & $36.5 \pm 0.3$ \\
\hline ETCO $_{2}(\mathrm{~mm} \mathrm{Hg})$ & $35.6 \pm 3.2$ & $36.2 \pm 3.5$ & $35 \pm 3.8$ \\
\hline Arterial lactate $(\mathrm{mmol} / \mathrm{L})$ & $1.1 \pm 0.13$ & $1.05 \pm 0.1$ & $1.08 \pm 0.16$ \\
\hline Arterial pH & $7.42 \pm 0.03$ & $7.41 \pm 0.04$ & $7.43 \pm 0.04$ \\
\hline EF (\%) & $71 \pm 8$ & $68 \pm 7$ & $70 \pm 7$ \\
\hline FS (\%) & $45 \pm 7$ & $43 \pm 6$ & $41 \pm 6$ \\
\hline Duration of PC (s) & & $251 \pm 31$ & $254 \pm 35$ \\
\hline
\end{tabular}

Table 1 Baseline and CPR characteristics of three groups

Values are presented as mean $\pm \mathrm{SD}$. $C P R$ cardiopulmonary resuscitation group, $C A$ cardiac arrest, EMP empagliflozin, MAP mean artery pressure, ETCO2 end-tidal $\mathrm{CO}_{2}, E F$ ejection fraction, $F S$ fractional shortening, $P C$ precordial compression

\section{Figures}

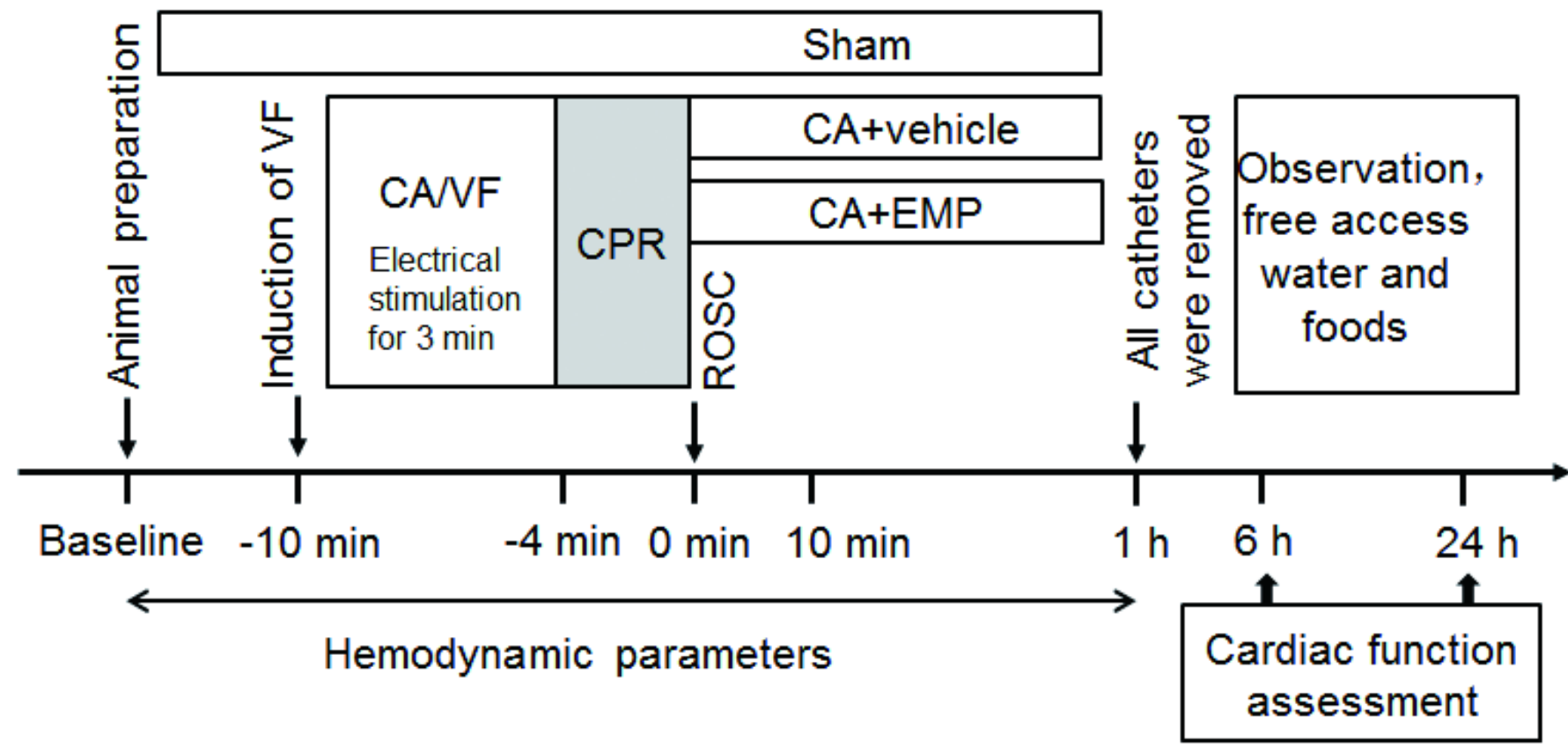

Figure 1 
Experimental procedures in VF-induced cardiac arrest model. VF ventricular fibrillation, CA cardiac arrest, CPR cardiopulmonary resuscitation, ROSC return of spontaneous circulation, EMP empagliflozin

a

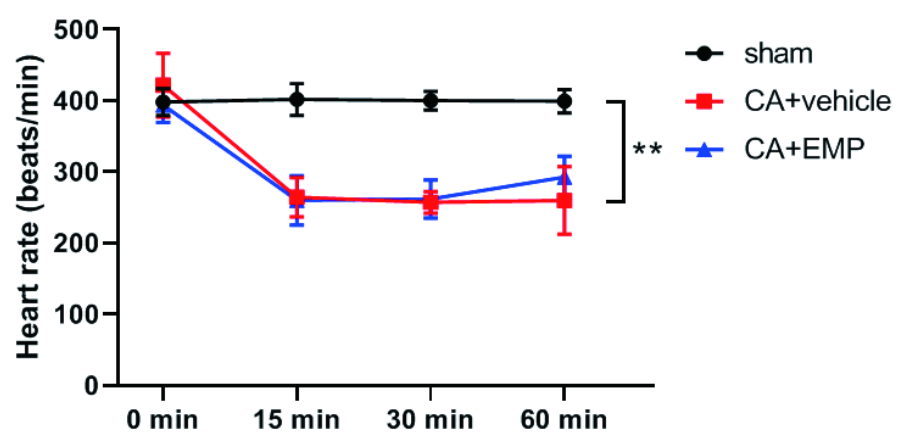

C

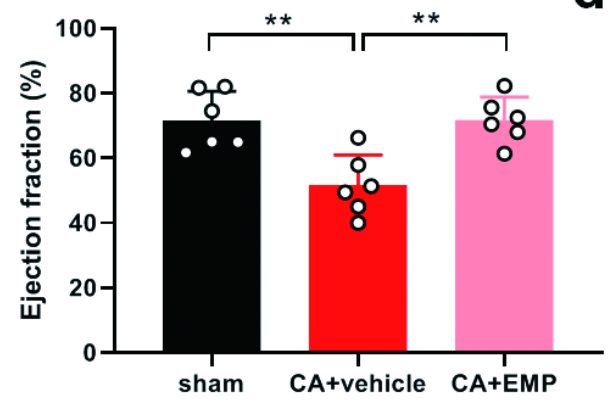

d

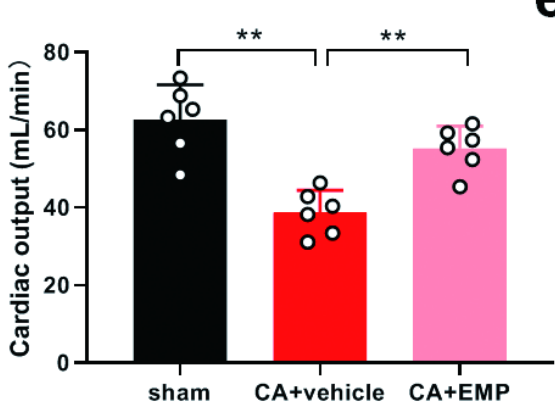

b

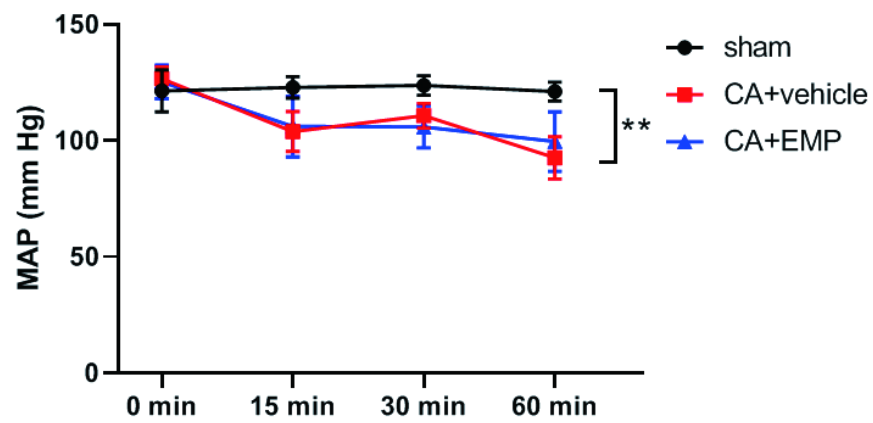

e

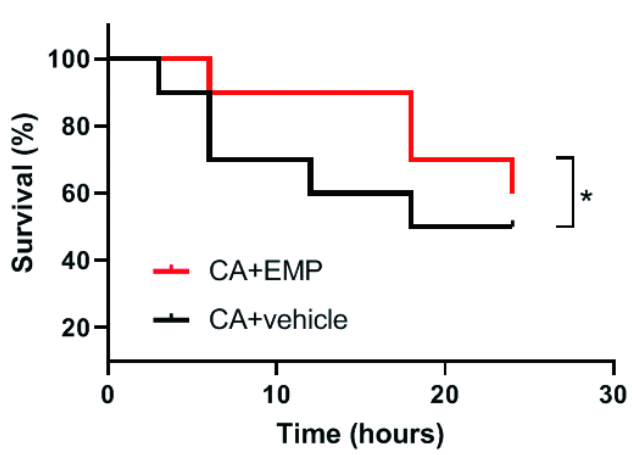

Figure 2

Effect of empagliflozin (EMP) on cardiac function after cardiac arrest in rats. a Effect of EMP on heart rate within 1 hour after return of spontaneous circulation (ROSC) ( $n=7$ to 10). $b$ Effect of EMP on mean artery pressure (MAP) within 1 hour after ROSC ( $n=7$ to 10). c EMP increased left ventricular ejection fraction at 24 hours after ROSC $(n=6)$. d EMP increased cardiac output at 24 hours after ROSC $(n=6)$. e EMP improved survival of the rats after cardiac arrest within 24 hours $(n=10)$. Data are presented as mean $\pm S D, * P<0.05, \star * P<0.01$. 

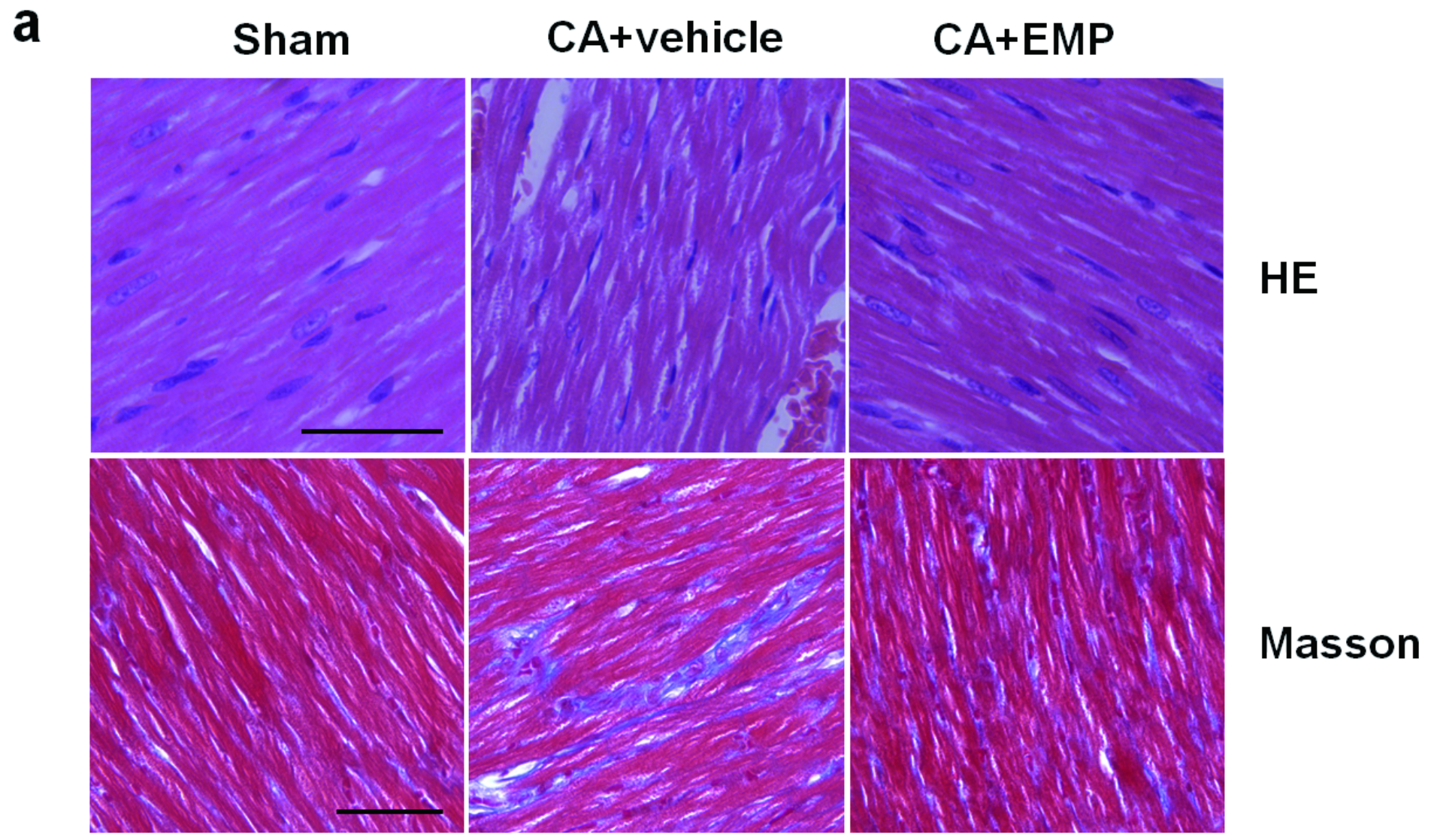

b

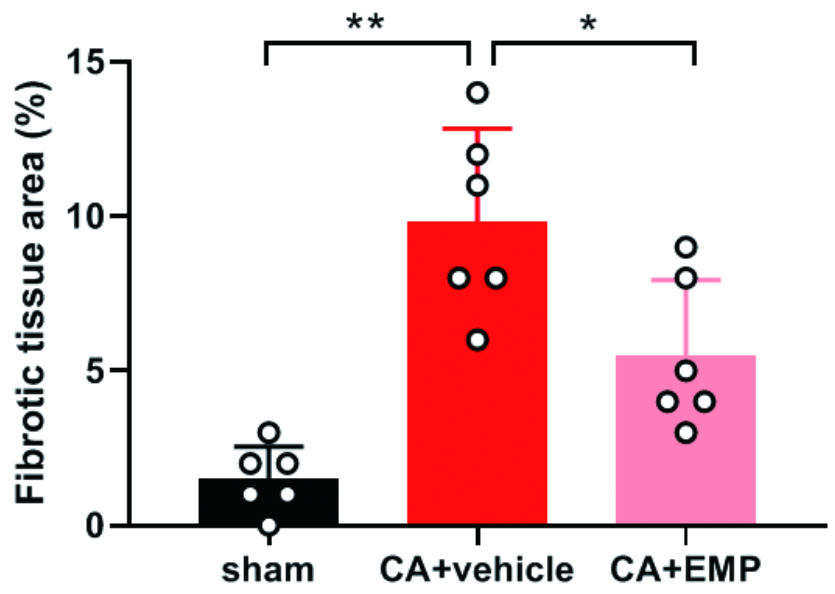

C

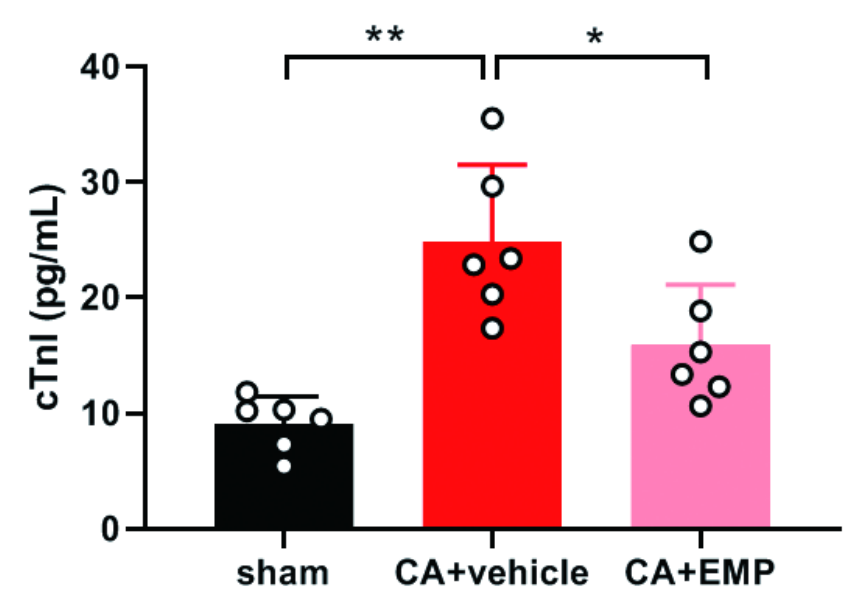

Figure 3

Effect of empagliflozin (EMP) on myocardial injury after cardiac arrest in rats. a Representative photographs of Hematoxylin and Eosin (HE) and Masson staining of heart sections obtained 24 hours after return of spontaneous circulation (ROSC). HE staining showed that EMP decreased myocardial necrosis after ROSC; Masson staining showed that EMP decreased myocardial fibrosis (stained blue) after ROSC. Scale bar $=50 \mu \mathrm{m}$. b Quantification of myocardial fibrotic area from Masson stained section. c EMP decreased serum cardiac troponin I (cTnl) level at 24 hours after ROSC. Data are presented as mean $\pm S D, n=6, * P<0.05$, ** $P<0.01$. 

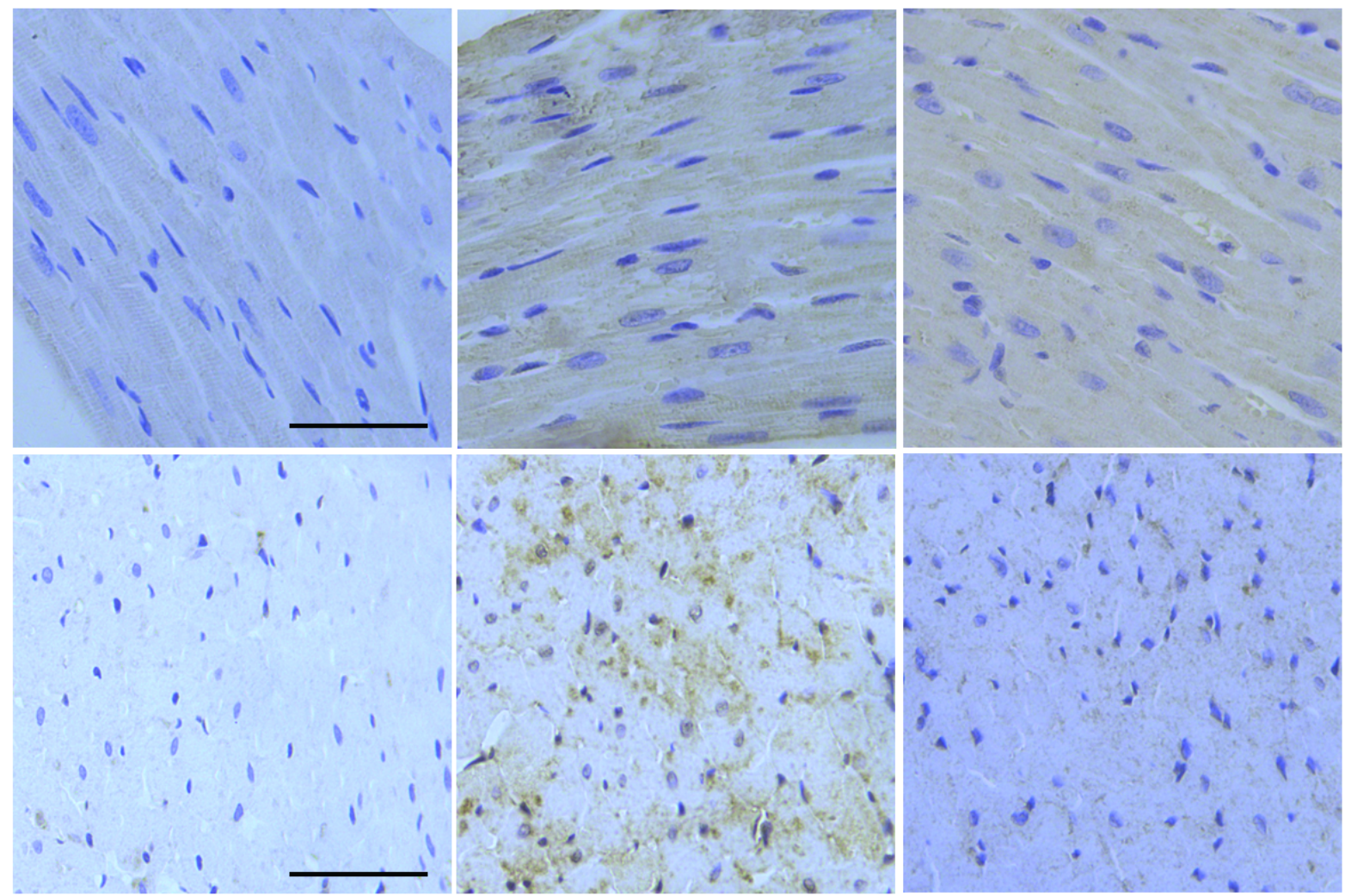

\section{4-HNE}

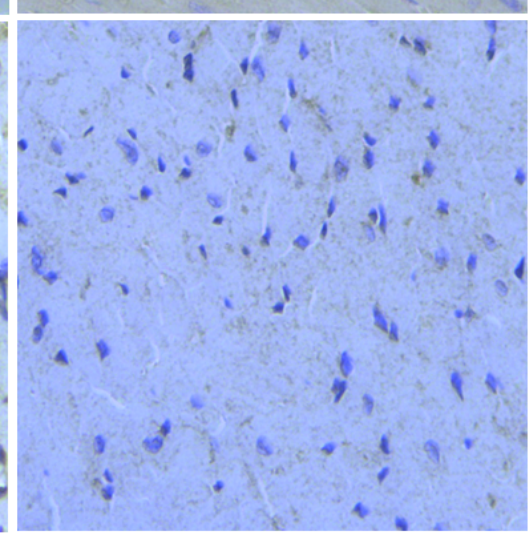

\section{8-OHdG}

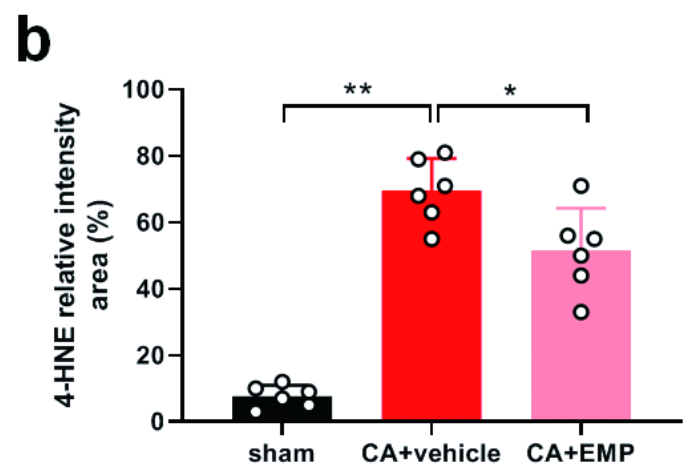

C

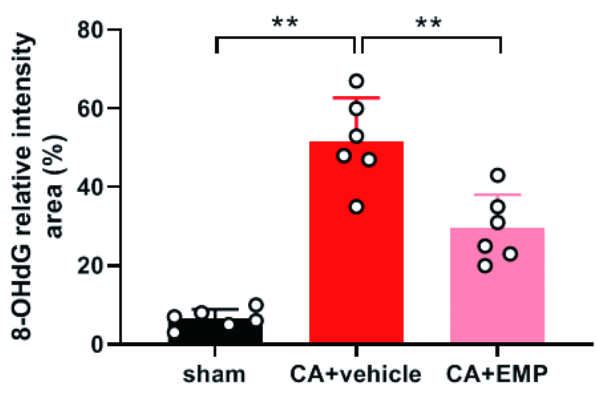

d

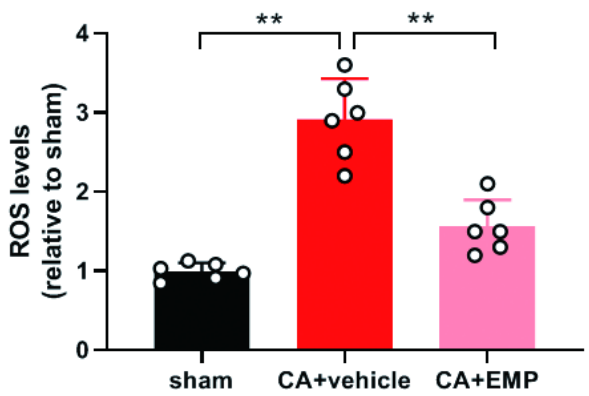

Figure 4

Empagliflozin (EMP) decreased myocardial oxidative stress after cardiac arrest in rats. a Representative photographs of 4-hydroxynonenal (4-HNE) and 8-hydroxy-2'-deoxyguanosine (8-OHdG) immunostaining of heart sections obtained 24 hours after return of spontaneous circulation (ROSC). Scale bar $=50 \mu \mathrm{m} . \mathrm{b}$ Quantification of 4-HNE staining levels from stained heart sections. c Quantification of 8-OHdG staining levels from stained heart sections. $d$ The relative reactive oxygen species (ROS) levels in heart tissues obtained 24 hours ROSC. Data are presented as mean \pm SD, $n=6,{ }^{*} P<0.05, * * P<0.01$. 
a

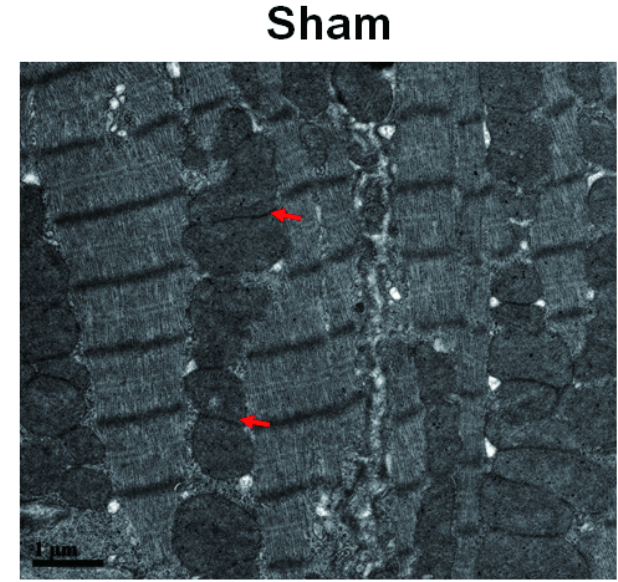

b

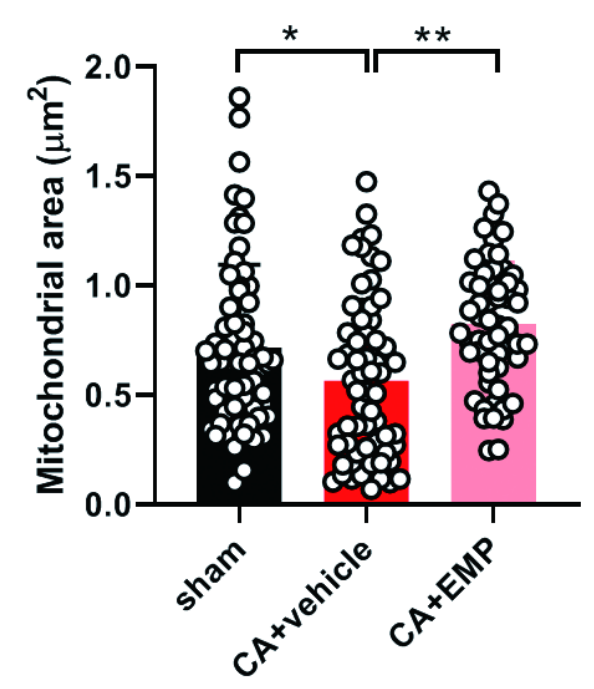

CA+vehicle

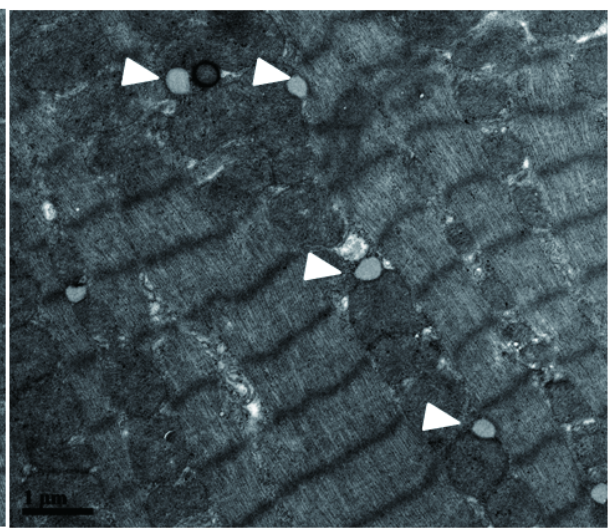

C

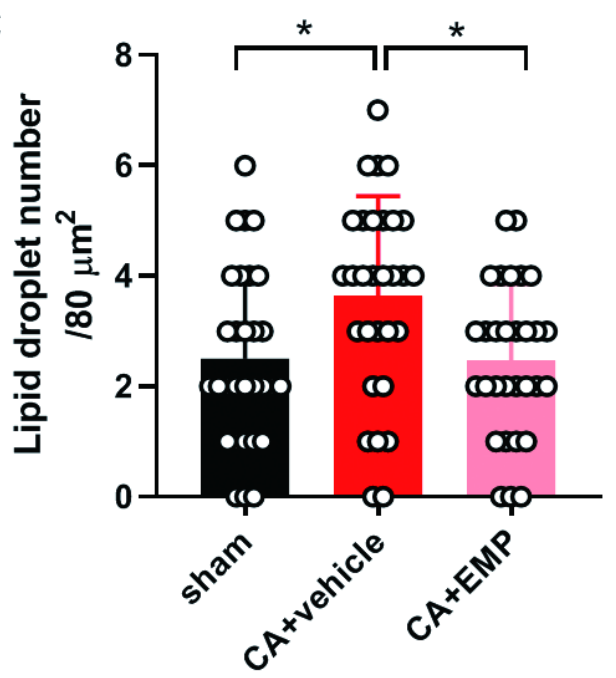

$\mathrm{CA}+\mathrm{EMP}$

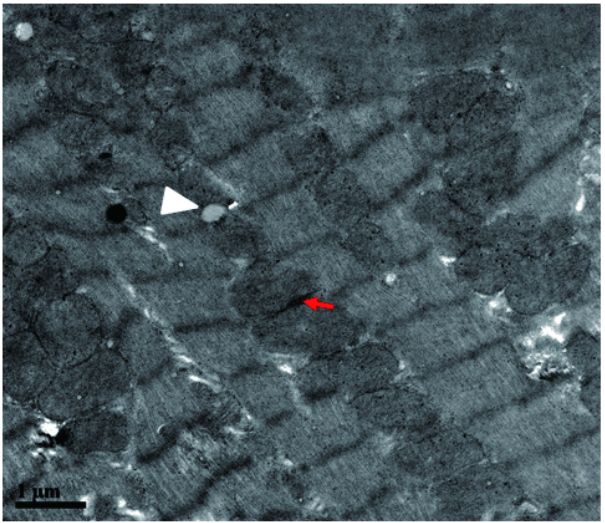

d

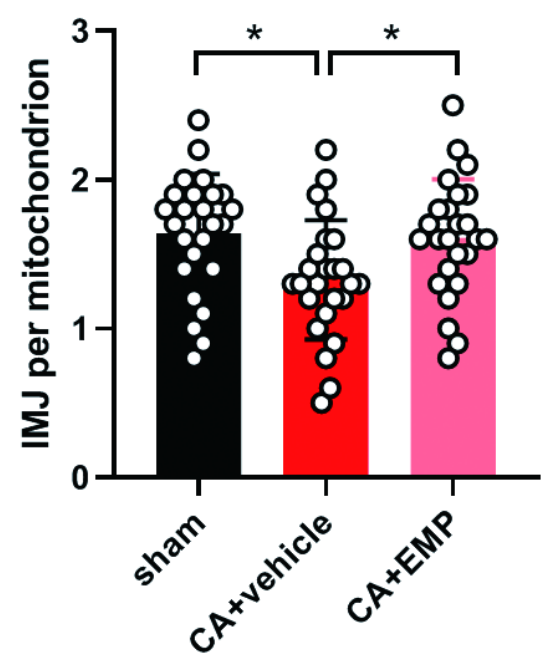

Figure 5

Effect of empagliflozin (EMP) on structural integrity of the myocardial mitochondria after cardiac arrest in rats. a Representative heart transmission electron microscopy (TEM) images of heart mitochondria at 24 hours after return of spontaneous circulation (ROSC). Arrow indicates inter-mitochondrial junctions (IMJs); Arrowhead indicates lipid droplet. Scale bar $=1 \mu \mathrm{m}$. b Quantification of heart mitochondrial area by TEM after ROSC. $\mathrm{n}=5$ and at least 60 mitochondria were counted in each group. c Quantification of heart mitochondrial lipid droplet content by TEM after ROSC. $n=5$ and 30 photographs were counted in each group. $d$ Quantification of heart IMJs by TEM after ROSC. $n=5$ and 25 photographs were counted in each group. Data are presented as mean $\pm S D$, $* P<0.05$, $* * P<0.01$. 
a

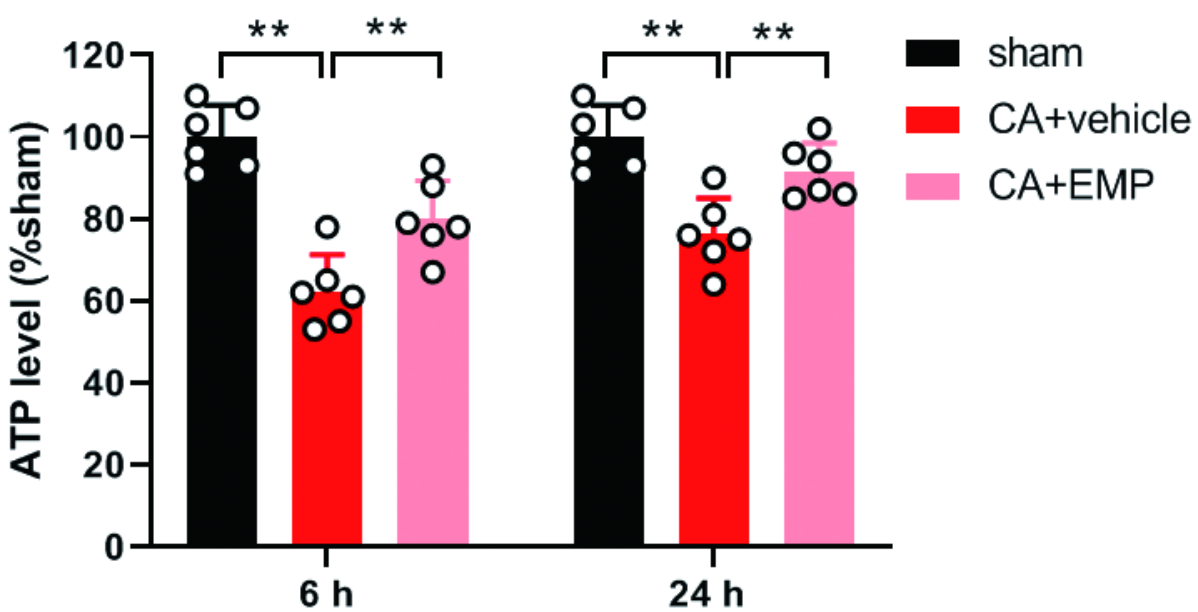

b

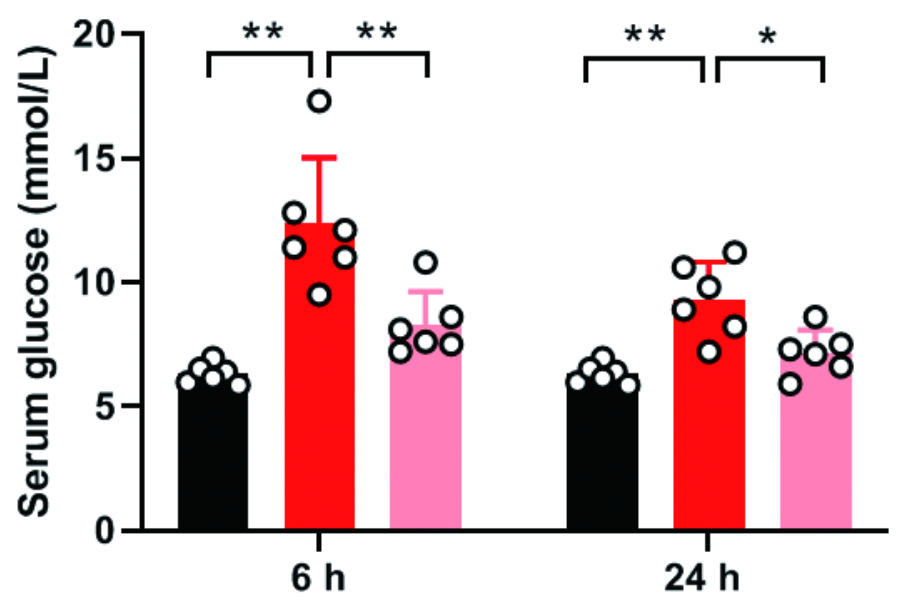

C

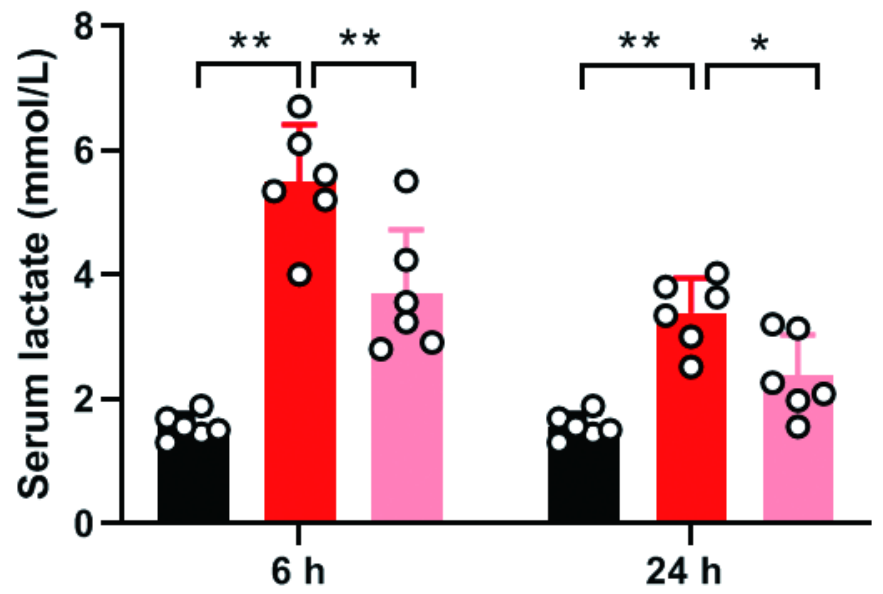

Figure 6

Empagliflozin (EMP) increased myocardial energy levels after cardiac arrest in rats. a EMP increased myocardial ATP levels at 6 and 24 hours after return of spontaneous circulation (ROSC). b EMP decreased serum glucose levels at 6 and 24 hours after ROSC. c EMP decreased serum lactate levels at 6 and 24 hours after ROSC. Data are presented as mean $\pm S D, n=6,{ }^{*}<0.05, \star \star P<0.01$. 
a

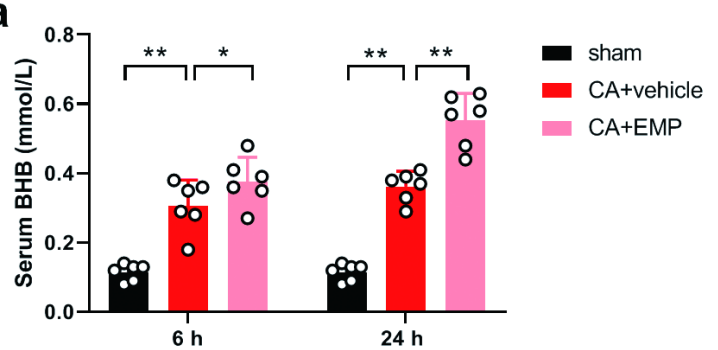

b

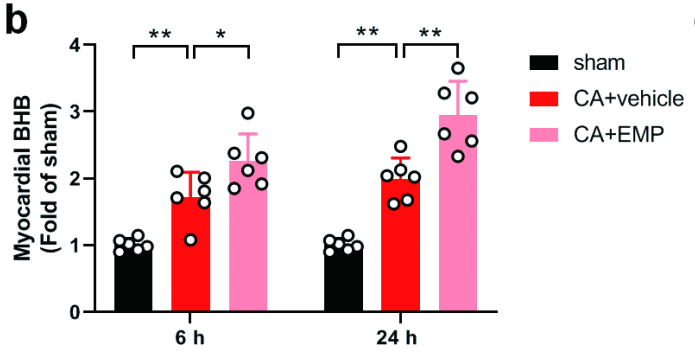

C

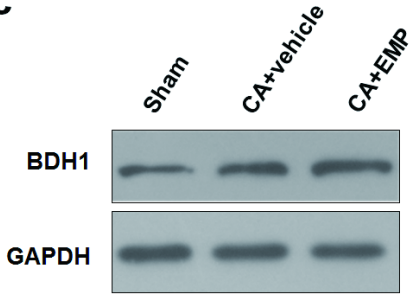

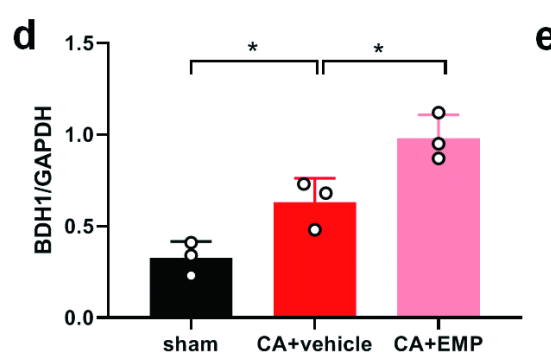
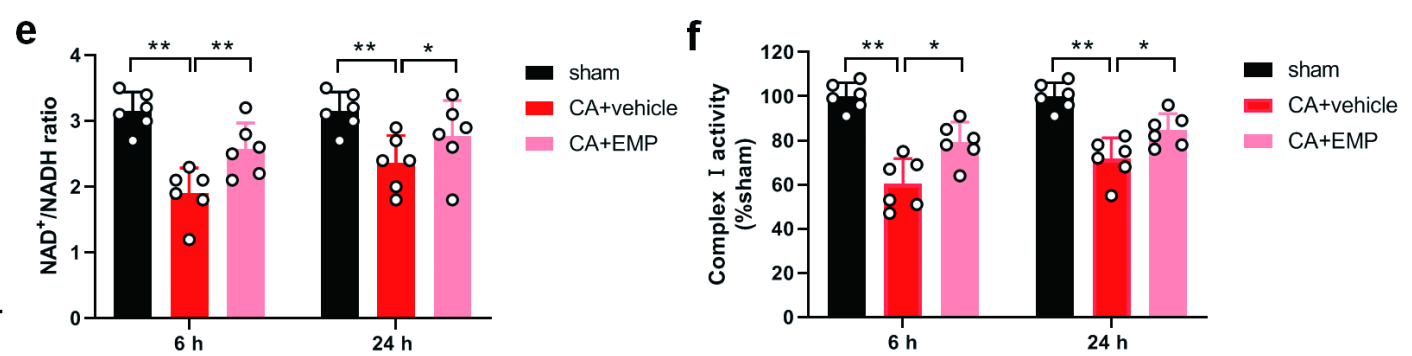

Figure 7

Empagliflozin (EMP) increased myocardial ketone metabolism and mitochondrial activity after cardiac arrest in rats. a EMP increased the serum $\beta$-hydroxybutyrate (BHB) levels at 6 and 24 hours after return of spontaneous circulation (ROSC). b EMP increased the myocardial BHB levels at 6 and 24 hours after ROSC. $c$ EMP increased the heart $\beta$-hydroxybutyrate dehydrogenase $(\mathrm{BDH} 1)$ protein expression at 24 hours after ROSC. GAPDH was used as the loading control. $d$ Quantification of heart BDH1 protein expression by western blot band intensity measurement. e EMP increased the myocardial NAD+/NADH

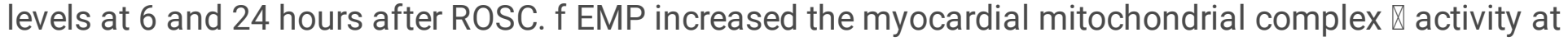
6 and 24 hours after ROSC. Data are presented as mean $\pm S D, n=3$ or $6,{ }^{*} P<0.05,{ }^{\star} \mathrm{P}<0.01$. 